\title{
ON THE PARTIAL SUMS OF HARMONIC DEVELOPMENTS AND OF POWER SERIES
}

\author{
BY \\ OTTO SZĀSZ
}

1. Introduction. Consider the class $E$ of power series $f(z)=\sum_{0}^{\infty} c_{\nu} z^{\nu}$, convergent for $|z|<1$ and such that $|f(z)| \leqq-1$. The following result is due to I. Schur and G. Szegö $[5]\left({ }^{1}\right)$.

For any series of the class $E$,

$$
\left|s_{n}(z)\right| \equiv\left|\sum_{0}^{n} c_{\nu} z^{\nu}\right| \leqq 1
$$

in $|z| \leqq r_{n}$, but not always in $|z|<r_{n}+\epsilon, \epsilon>0$, where $r_{n}$ is the largest $r$ for which

$$
T_{n}(r, \theta)=\frac{1}{2}+\sum_{1}^{n} r^{\nu} \cos \nu \theta \geqq 0 \quad \text { for all } \theta .
$$

The $r_{n}$ are non-decreasing,

$$
\begin{array}{lr}
r_{n}>1-\frac{\log 2 n}{n}, & n=1,2,3, \cdots, \\
r_{n}=1-\frac{\log 2 n-\log \log 2 n+\epsilon_{n}}{n}, & \lim _{n \rightarrow \infty} \epsilon_{n}=0 .
\end{array}
$$

We obtain the same constant $r_{n}$ if we assume $R f(z) \geqq 0$ and require $R s_{n}(z) \geqq 0$. Here $R u$ means the real part of $u$; $I u$ will denote the imaginary part.

In what follows, we consider harmonic sine developments

$$
H(r, \theta)=\sum_{1}^{\infty} b_{\nu} r^{v} \sin \nu \theta
$$

convergent for $0<r<1$, and non-negative for $0<\theta<\pi$. Evidently there exists an $R_{n}$ with the following properties:

(a) Whenever

$$
H(r, \theta) \geqq 0, \quad 0<r<1 ; 0<\theta<\pi,
$$

then,

$$
s_{n}(r, \theta) \equiv \sum_{1}^{n} b_{\nu} r^{\nu} \sin \nu \theta \geqq 0,0<r \leqq R_{n} ; 0<\theta<\pi .
$$

Presented to the Society, January 1, 1941; received by the editors March 10, 1941, and in revised form, May 7, 1941. The author is indebted to the referee for valuable suggestions.

(1) Numbers in brackets refer to the literature at the end of this paper. 
(b) For any $\epsilon>0$ we can find an $H$ satisfying (1.1) and such that $s_{n}(r, \theta)$ becomes negative for some $\theta$ and some $r<R_{n}+\epsilon$.

We denote the class of harmonic functions satisfying (1.1) by $T$. On writing $f(z)=\sum_{1}^{\infty} b_{\nu} z^{\nu}$, the power series $f(z)$ is regular in $|z|<1$, has all its coefficients real, and $I f(z) \geqq 0$ in $|z|<1, I z>0$. The class $T$ has been discussed by Rogosinski [4]; the function $f(z)$ is called typically real. (Cf. also S. Mandelbrojt [2].)

One of the results of the present paper is

$$
R_{n}=1-\frac{3 \log n}{n}+\frac{\log \log n}{n}+O(1 / n), \quad \text { as } n \rightarrow \infty .
$$

M. S. Robertson [3] gave the erroneous estimate

$$
R_{n} \geqq 1-2 \log n / n \quad \text { for } n>12 .
$$

His calculation yields however, as is seen easily, $R_{n} \geqq 1-4 \log n / n$, for $n>n_{0}\left({ }^{2}\right)$. We then apply the properties of $R_{n}$ to Fourier series of convex functions and to a certain class of power series.

Note that if $\phi(\theta) \sim \sum_{1}^{\infty} b_{\nu} \sin \nu \theta, \phi(\theta) \geqq 0,0<\theta<\pi$, then

$$
\begin{aligned}
H(r, \theta) & =\frac{2}{\pi} \int_{0}^{\pi} \phi(x)\left(\sum_{1}^{\infty} r^{\nu} \sin \nu \theta \sin \nu x\right) d x \\
& =\frac{1}{\pi} \int_{0}^{\pi} \phi(x) \sum_{1}^{\infty} r^{\nu}[\cos \nu(\theta-x)-\cos \nu(\theta+x)] d x \\
& =\frac{1-r^{2}}{2 \pi} \int_{0}^{\pi} \phi(x)\left(\frac{1}{1-2 r \cos (\theta-x)+r^{2}}-\frac{1}{1-2 r \cos (\theta+x)+r^{2}}\right) d x \\
& =\frac{2 r\left(1-r^{2}\right)}{\pi} \sin \theta \int_{0}^{\pi} \frac{\phi(x) \sin x d x}{\left[1-2 r \cos (\theta-x)+r^{2}\right]\left[1-2 r \cos (\theta+x)+r^{2}\right]}
\end{aligned}
$$

Hence $H(r, \theta)$ belongs to the class $T$. (Cf. Zygmund [8, p. 57].)

2. Characterization of $R_{n}$. We quote the following lemma, due to Fejér (Turán [7]).

LEMMA 1. In order that

$$
\sum_{\nu=1}^{n} \lambda, \sin \nu x \sin \nu y \geqq 0 \text { for } 0<x<\pi, 0<y<\pi,
$$

it is necessary and sufficient that

$$
\sum_{1}^{n} \nu \lambda_{\nu} \sin \nu \theta \geqq 0 \quad \text { for } 0<\theta<\pi .
$$

(2) Robertson, Annals of Mathematics, (2), vol. 42 (1941), pp. 829-838. 
We now prove

THEOREM 1. The quantity $R_{n}$ as defined in $\$ 1$ is the largest $r$ for which

$$
S_{n}(r, \theta) \equiv \sum_{1}^{n} \nu r^{\nu} \sin \nu \theta \geqq 0 \quad \text { for } 0<\theta<\pi .
$$

We have for $0<\rho<1$,

$$
\rho^{\nu} b_{\nu}=\frac{2}{\pi} \int_{0}^{\pi} H(\rho, x) \sin \nu x d x, \quad \nu=1,2,3, \cdots
$$

hence

$$
s_{n}(r, \theta)=\frac{2}{\pi} \int_{0}^{\pi} H(\rho, x)\left(\sum_{1}^{n}\left(\frac{r}{\rho}\right)^{\nu} \sin \nu \theta \sin \nu x\right) d x .
$$

For any $r<R_{n}$ we can choose $\rho<1$ so that $r / \rho<R_{n}$; we then obtain by Lemma 1 (for $\left.\lambda_{\nu}=r^{\nu}\right) s_{n}(r, \theta) \geqq 0$ for any $r<R_{n}$ and for $0<\theta<\pi$; hence (a) holds for $r \leqq R_{n}$. Conversely, for the function

$$
H(r, \theta)=\sum_{1}^{\infty} \nu r^{\nu} \sin \nu \theta=r \sin \theta \frac{1-r^{2}}{\left(1-2 r \cos \theta+r^{2}\right)^{2}}>0
$$

the function

$$
s_{n}(r, \theta)=\sum_{1}^{n} \nu r^{\nu} \sin \nu \theta
$$

becomes negative for any $r>R_{n}$ and for some $\theta$ in $(0, \pi)$. This proves Theorem 1 . To estimate $R_{n}$ we first give another characterization for it. An easy calculation yields

$$
\begin{aligned}
& \frac{\left(1-2 r \cos \theta+r^{2}\right)^{2}}{r \sin \theta} \sum_{1}^{n} \nu r^{\nu} \sin \nu \theta \\
& =1-r^{2}-(n+1) r^{n+2} \cdot \frac{\sin (n-1) \theta}{\sin \theta}+r^{n+1}\left(2 n+2+n r^{2}\right) \frac{\sin n \theta}{\sin \theta} \\
& \quad-\quad r^{n}\left(n+1+2 n r^{2}\right) \frac{\sin (n+1) \theta}{\sin \theta}+n r^{n+1} \frac{\sin (n+2) \theta}{\sin \theta} \\
& \equiv C_{n}(r, \theta) .
\end{aligned}
$$

This furnishes

THEOREM 2. $R_{n}$ is the largest $r$ for which

$$
C_{n}(r, \theta) \geqq 0
$$

for all $\theta$.

Evidently 


$$
\begin{aligned}
C_{n}(r, \pi)= & 1-r^{2}+\left(n^{2}-1\right) r^{n+2}(-1)^{n-1}+n r^{n+1}\left(2 n+2+n r^{2}\right)(-1)^{n-1} \\
& +(n+1) r^{n}\left(n+1+2 n r^{2}\right)(-1)^{n-1}+n(n+2) r^{n+1}(-1)^{n-1} \\
= & 1-r^{2}+(-1)^{n-1}\left\{\left(n^{2}-1\right) r^{n+2}+n r^{n+1}\left(2 n+2+n r^{2}\right)\right. \\
& \left.\quad+(n+1) r^{n}\left(n+1+2 n r^{2}\right)+n(n+2) r^{n+1}\right\} .
\end{aligned}
$$

Thus

$$
\begin{aligned}
& C_{n}(r, \theta) \geqq 1-r^{2}-\left\{\left(n^{2}-1\right) r^{n+2}+n r^{n+1}\left(2 n+2+n r^{2}\right)\right. \\
& \left.+(n+1) r^{n}\left(n+1+2 n r^{2}\right)+n(n+2) r^{n+1}\right\}, \quad n \geqq 1,
\end{aligned}
$$

and equality holds if $n=2 k$, and $\theta=\pi$. This yields

Theorem 3. Denote the unique positive root of the equation

$p_{n}(r) \equiv 1-r^{2}-(n+1)^{2} r^{n}-n(3 n+4) r^{n+1}-\left(3 n^{2}+2 n-1\right) r^{n+2}-n^{2} r^{n+3}=0$

by $\rho_{n}$. Then $R_{n} \geqq \rho_{n}$, and equality holds for $n=2 k, k \geqq 1$.

Note that $p_{n}(0)=1, p_{n}(1)<0, p_{n}^{\prime}(r)<0$. Hence $\rho_{n}$ is unique and

$$
0<\rho_{n}<1 \text {. }
$$

Evidently $p_{n}(-1)=0$, hence $1+r$ can be factored out, and we get

$$
\text { (2.3) } \frac{p_{n}(r)}{1+r}=1-r-(n+1)^{2} r^{n}-\left(2 n^{2}+2 n-1\right) r^{n+1}-n^{2} r^{n+2} \equiv q_{n}(r) \text {, }
$$

so that $q_{n}\left(\rho_{n}\right)=0$.

3. Estimation of $\rho_{n}$ and $R_{n}$. Direct calculation gives

$$
R_{1}=1 ; \rho_{1}=0.182 \cdots \text {. }
$$

Also $\rho_{2}=R_{2}$, and

$$
S_{2}(r, \theta)=r \sin \theta+2 r^{2} \sin 2 \theta=r \sin \theta(1+4 r \cos \theta),
$$

which yields by Theorem 1: $R_{2}=1 / 4=\rho_{2}$. A similar calculation yields $R_{3}=2^{1 / 2} / 3$.

We shall prove

$$
\rho_{n}=1-\frac{3 \log n}{n}+\frac{\log \log n+\log 3 / 4+\epsilon_{n}}{n}, \quad \epsilon_{n} \rightarrow 0 \text { as } n \rightarrow \infty .
$$

Let $c$ be a constant, and

$$
r_{n}(c)=1-\frac{3 \log n}{n}+\frac{\log \log n+c}{n} ;
$$

then from

$$
\log (1-x)=-x+O\left(x^{2}\right) \quad \text { as } x \rightarrow 0,
$$


we conclude

$$
\begin{aligned}
\left\{r_{n}(c)\right\}^{n} & =\exp \left\{-3 \log n+\log \log n+c+O\left(n^{-1} \log ^{2} n\right)\right\} \\
& =n^{-3} \log n \cdot e^{c}\left\{1+O\left(n^{-1} \log ^{2} n\right)\right\} \text { as } n \rightarrow \infty
\end{aligned}
$$

Furthermore, from (2.3), (3.2), and (3.3)

$$
q_{n}\left\{r_{n}(c)\right\}=\frac{3 \log n}{n}-\frac{\log \log n+c}{n}-\frac{4 \log n}{n} \cdot \epsilon^{c}\{1+o(1)\},
$$

hence

$$
\frac{n q_{n}\left\{r_{n}(c)\right\}}{\log n} \rightarrow 3-4 e^{c}
$$

Thus for

$$
c=\log 3 / 4+\epsilon,
$$

$\boldsymbol{\epsilon}$ a given small number, and for sufficiently large values of $n$

$$
\operatorname{sgn} q_{n}\left\{r_{n}(c)\right\}=\operatorname{sgn} \epsilon,
$$

from which follows (3.1).

We have thus proved

Theorem 4. If $\rho_{n}>0$ and $p_{n}\left(\rho_{n}\right)=0$, then

$$
\rho_{n}=1-\frac{3 \log n}{n}+\frac{\log \log n+\log 3 / 4+\epsilon_{n}}{n}, \quad \text { where } \epsilon_{n} \rightarrow 0 \text { as } n \rightarrow \infty .
$$

4. Derivation of an asymptotic estimate for $R_{n}$. On writing

$$
R_{n}=1-\frac{3 \log n}{n}+\frac{\log \log n+\delta_{n}}{n}, \quad n>1,
$$

it follows from Theorem 3 that

$$
\delta_{n} \geqq \log 3 / 4+\epsilon_{n},
$$

and equality holds for $n=2 k, k \geqq 1$; hence from Theorem 4

$$
\liminf _{n \rightarrow \infty} \delta_{n}=\log 3 / 4, \quad \lim _{k \rightarrow \infty} \delta_{2 k}=\log 3 / 4 \text {. }
$$

It remains to give an estimate for $R_{2 k-1}$ from above.

If for a particular value of $\theta$ and $r, C_{2 k-1}(r, \theta)<0$, then by Theorem 2, evidently $R_{2 k-1}<r$. We now choose $\theta=\pi-(3 \pi / 4 k)$; then 


$$
\begin{aligned}
C_{2 k-1}(r, \theta)= & -r^{2}+\frac{1}{\sin (3 \pi / 4 k)}\left\{2 k r^{2 k+1} \sin \frac{3(k-1) \pi}{2 k}\right. \\
& +r^{2 k}\left[4 k+(2 k-1) r^{2}\right] \sin \frac{3(2 k-1) \pi}{4 k} \\
& +r^{2 k-1}\left[2 k+2(2 k-1) r^{2}\right] \sin \frac{3 \pi}{2} \\
& \left.+(2 k-1) r^{2 k} \sin \frac{3(2 k+1) \pi}{4 k}\right\} \\
= & 1-r^{2}-\frac{1}{\sin (3 \pi / 4 k)}\left\{2 k r^{2 k+1} \cos \frac{3 \pi}{2 k}\right. \\
& +r^{2 k}\left[4 k+(2 k-1) r^{2}\right]\left[\cos \frac{3 \pi}{4 k}\right] \\
& \left.+2 k r^{2 k-1}+(4 k-2) r^{2 k+1}+(2 k-1) r^{2 k} \cos \frac{3 \pi}{4 k}\right\} \\
< & 1-r^{2}-\frac{4 k}{3 \pi}\left\{2 k r^{2 k+1}\left(1-\frac{9 \pi^{2}}{8 k^{2}}\right)\right. \\
& +r^{2 k}\left[4 k+(2 k-1) r^{2}\right]\left(1-\frac{9 \pi^{2}}{32 k^{2}}\right) \\
& \left.+2 k r^{2 k-1}+(4 k-2) r^{2 k+1}+(2 k-1) r^{2 k}\left(1-\frac{9 \pi^{2}}{32 k^{2}}\right)\right\}, k \geqq 3
\end{aligned}
$$

(since $\cos x>1-x^{2} / 2$ for all $x$ ). Hence

$$
\begin{aligned}
C_{2 k-1}(r, \theta)< & 1-r^{2}-(2 k / 5)\left\{k r^{2 k+1}+2 k r^{2 k}+(k-1 / 2) r^{2 k+2}+2 k r^{2 k-1}\right. \\
& \left.+(4 k-2) r^{2 k+1}+(k-1 / 2) r^{2 k}\right\}, \quad k \geqq 5,
\end{aligned}
$$

thus

$$
C_{2 k-1}(r, \theta)<1-r^{2}-(2 k / 5)(11 k-3) r^{2 k+2}<1-r^{2}-4 k^{2} r^{2 k+2} .
$$

Choosing $r$ so that

$$
1-r^{2}-4 k^{2} r^{2 k+2} \leqq 0,
$$

we get

$$
C_{2 k-1}(r, \theta)<0, \quad R_{2 k-1}<r .
$$

To find an upper bound for $r$, we put

$$
r=1-\frac{3 \log (2 k-1)}{2 k-1}+\frac{\log \log (2 k-1)+c}{2 k-1}
$$


we obtain as in (3.3)

$$
\begin{aligned}
r^{2 k-1} & =\exp \left\{-3 \log (2 k-1)+\log \log (2 k-1)+c+O\left(k^{-1} \log ^{2} k\right)\right\} \\
& =(2 k-1)^{-3} \log (2 k-1) \cdot e^{c}\left\{1+O\left(k^{-1} \log ^{2} k\right)\right\} .
\end{aligned}
$$

Thus, using (4.2),

$$
\begin{aligned}
\frac{4 k^{2} r^{2 k+2}}{1-r^{2}} & =\left(\frac{2 k}{2 k-1}\right)^{2} \cdot \frac{r^{3}}{1+r} \cdot \frac{r^{2 k-1}(2 k-1)^{2}}{1-r} \\
& =\frac{1+o(1)}{2+o(1)} \cdot \frac{1}{3} e^{c}\{1+o(1)\} \rightarrow \frac{1}{6} e^{c} \quad \text { as } k \rightarrow \infty .
\end{aligned}
$$

Hence (4.1) is satisfied for all sufficiently large $k$ provided $e^{c} / 6>1$, that is, $c>\log 6$. It now follows that $\lim \sup _{k \rightarrow \infty} \delta_{2 k-1} \leqq 6$. Summarizing we have

THEOREM 5. Let

$$
R_{n}=1-\frac{3 \log n}{n}+\frac{\log \log n+\delta_{n}}{n}, \quad n>1 ;
$$

then $\lim _{k \rightarrow \infty} \delta_{2 k}=\log 3 / 4$, and

$\log 3 / 4 \leqq \liminf _{k \rightarrow \infty} \delta_{2 k-1} \leqq \limsup _{k \rightarrow \infty} \delta_{2 k-1} \leqq 6$.

5. Application to Fourier series. Consider the roof-function

$$
\frac{2 b}{a(\pi-a)} \sum_{1}^{\infty} \frac{\sin \nu a \sin \nu \theta}{\nu^{2}}= \begin{cases}\frac{b \theta}{a} & \text { for } 0 \leqq \theta \leqq a, \\ b \frac{\pi-\theta}{\pi-a} & \text { for } a \leqq \theta \leqq \pi,\end{cases}
$$

where $0<a<\pi, 0<b$, and the corresponding harmonic function

$$
\frac{2 b}{a(\pi-a)} \sum_{1}^{\infty} r^{\nu} \frac{\sin \nu a \sin \nu \theta}{\nu^{2}}=H(r ; a, b) \text {. }
$$

Denote its partial sums by

$$
H_{n}(r, \theta)=\frac{2 b}{a(\pi-a)} \sum_{1}^{n} r^{\nu} \frac{\sin \nu a \sin \nu \theta}{\nu^{2}} ;
$$

then

$$
\frac{\partial^{2} H_{n}(r, \theta)}{\partial \theta^{2}}=-\frac{2 b}{a(\pi-a)} \sum_{1}^{n} r^{\nu} \sin \nu a \sin \nu \theta \leqq 0
$$

for $0<r \leqq R_{n}, 0<\theta<\pi$, by Lemma 1 and Theorem 1 . Hence $H_{n}(r, \theta)$ is con- 
vex upwards for $0<\theta<\pi, r \leqq R_{n}$; but not convex for $r>R_{n}$. The same is true for the limiting cases $a \rightarrow 0$ and $a \rightarrow \pi$. In which cases

$$
\begin{aligned}
& H(r ; 0, b)=\frac{2 b}{\pi} \sum_{1}^{\infty} r^{\nu} \frac{\sin \nu \theta}{\nu}, \\
& H(r ; \pi, b)=\frac{2 b}{\pi} \sum_{1}^{\infty} r^{\nu} \frac{\sin \nu(\pi-\theta)}{\nu} .
\end{aligned}
$$

Moreover every polygon convex upwards and lying above the axis of abscissae is expressible as a finite sum with positive coefficients of roof-functions. Hence the partial sums of the corresponding harmonic development are convex upwards for $r \leqq R_{n}$. Finally any function positive in $0<\theta<\pi$, and convex upwards can be approximated uniformly by such polygons; hence we have

THEOREM 6. If $f(\theta)>0$ in $0<\theta<\pi$, and is convex upwards, and if $f(\theta) \sim \sum_{1}^{\infty} b_{\nu} \sin \nu \theta$, then $\sum_{1}^{n} r^{\nu} b_{\nu} \sin \nu \theta$ is convex upwards in $0<\theta<\pi, r \leqq R_{n}$; but not always for $r<R_{n}+\epsilon, \epsilon>0$.

6. Cosine series. We now consider the cosine series of the step function

$$
\frac{2 b}{\pi}\left\{\frac{\pi-a}{2}-\sum_{1}^{\infty} \frac{\sin \nu a \cos \nu \theta}{\nu}\right\}=\left\{\begin{array}{lll}
0 & \text { for } & 0 \leqq \theta<a, \\
b & \text { for } & a<\theta \leqq \pi
\end{array}\right.
$$

where $0<a<\pi, b>0$; and the corresponding harmonic development

$$
K(r, \theta)=\frac{b}{\pi}(\pi-a)-\frac{2 b}{\pi} \sum_{1}^{\infty} r^{\nu} \frac{\sin \nu a \cos \nu \theta}{\nu} .
$$

For the partial sums $K_{n}(r, \theta)$ of this series we have

$$
\frac{\partial K_{n}(r, \theta)}{\partial \theta}=\frac{2 b}{\pi} \sum_{1}^{n} r^{\nu} \sin \nu a \sin \nu \theta \geqq 0 \quad \text { for } 0<r \leqq R_{n}, 0<\theta<\pi ;
$$

hence $K_{n}(r, \theta)$ is monotonic increasing in the same domain; $R_{n}$ cannot be replaced by $R_{n}+\epsilon, \epsilon>0$. The same statement for any monotonic increasing function follows now in an obvious way. Hence we have

THEOREM 7. If $f(\theta)$ is monotonic in $0<\theta<\pi$, and

$$
f(\theta) \sim a_{0} / 2+\sum_{1}^{\infty} a_{\nu} \cos \nu \theta,
$$

then the nth partial sum of $a_{0} / 2+\sum_{1}^{\infty} a_{\nu} r^{\nu} \cos \nu \theta$ is monotonic in the same sense for $0<r \leqq R_{n}$, and here $R_{n}$ cannot be replaced by $R_{n}+\epsilon, \epsilon>0$.

7. Curves convex in direction of the $v$-axis. We say that a curve in the $(u, v)$-plane is convex in the direction of the $v$-axis if any parallel to the $v$-axis 
has at most two points in common with the curve. This class of mappings was considered by L. Fejér [1] and the author [6]. We now prove

THEOREM 8. Suppose the power series $\sum_{0}^{\infty} a_{\nu} z^{\nu}=f(z)=w=u+i v$ is regular in $|z|<1$, and all $a_{\nu}$ are real: Suppose further that the images $K_{r}$ of the circles $|z|=r, 0<r<1$, are convex in the direction of the v-axis (thus $f(z)$ is univalent). Then the partial sum $\sum_{0}^{n} a_{\nu} z^{\nu}$ has the same property in $|z| \leqq R_{n}$, but-in generalnot in a larger circle.

For the proof we may assume without loss of generality that the upper half of the circle $|z|<1$ is mapped onto the upper half of the image in the $w$-plane. On writing $w\left(e^{i \theta}\right)=u(\theta)+i v(\theta) \sim \sum_{0}^{\infty} a_{\nu} \cos \nu \theta+i \sum_{0}^{\infty} a_{\nu} \sin \nu \theta$, we find that $v(\theta)$ is positive for $0<\theta<\pi$, and (from the assumption) $u(\theta)$ is decreasing in the same interval. Our theorem follows now from Theorems 5 and 7 .

8: Conclusion. Suppose $f(z)=\sum_{1}^{\infty} b_{\nu} z^{\nu}$ is a typically real function, that is,

$$
\sum_{1}^{\infty} b_{\nu} r^{\nu} \sin \nu \theta \geqq 0 \quad \text { for } \quad 0<r<1,0<\theta<\pi \text {. }
$$

Then the Riesz means of second order

$$
P_{n}(z)=(n+1)^{-2} \sum_{\nu=1}^{n}(n-\nu+1)^{2} b_{\nu} z^{\nu}, \quad n \geqq 1,
$$

are typically real in $|z| \leqq 1$ (Szász [6]; cf. Theorem 1). Evidently $\lim _{n \rightarrow \infty} P_{n}(z)$ $=f(z)$ in $|z|<1$, uniformly in $|z| \leqq r, r<1$. Another such sequence of polynomials is

$$
s_{n}\left(R_{n} z\right)=\sum_{\nu=1}^{n} b_{\nu} R_{n}^{\prime} z^{\prime}, \quad n \geqq 1 .
$$

These polynomials are typical real in $|z| \leqq 1$ by property (a) of $\S 1$. Furthermore for $|z| \leqq r<1$

$$
\begin{aligned}
\left|f(z)-s_{n}\left(R_{n} z\right)\right| & \leqq \sum_{1}^{n}\left|b_{\nu}\right| r^{\nu}\left(1-R_{n}^{\nu}\right)+\sum_{n+1}^{\infty}\left|b_{\nu}\right| r^{\nu} \\
& <\left(1-R_{n}\right) \sum_{1}^{n} \nu\left|b_{\nu}\right| r^{\nu}+\sum_{n+1}^{\infty}\left|b_{\nu}\right| r^{\nu} \rightarrow 0, \text { as } n \rightarrow \infty
\end{aligned}
$$

Hence

uniformly in $|z| \leqq r<1$.

$$
\lim _{n \rightarrow \infty} s_{n}\left(R_{n} z\right)=f(z)
$$

\section{REFERENCES}

1. L. Fejér, Neue Eigenschaften der Mittelwerte bei den Fourierreihen, Journal of the London Mathematical Society, vol. 8 (1933), pp. 53-62. 
2. S. Mandelbrojt, Quelques remarques sur les fonctions univalentes, Bulletin des Sciences Mathématiques, (2), vol. 58 (first part) (1934), pp. 185-200.

3. M.S. Robertson, On the theory of univalent functions, Annals of Mathematics, (2), vol. 37 (1936), pp. 374-408.

4. W. Rogosinski, Über positive harmonische Entwicklungen und typischreelle Potenzreihen, Mathematische Zeitschrift, vol. 35 (1932), pp. 93-121.

5. I. Schur and G. Szegö, Über die Abschnitte einer im Einheitskreise beschränkten Potenzreihe, Sitzungsberichte der Preussischen Akademie, 1923, pp. 545-560.

6. O. Szász, On the Cesàro and the Riesz means of Fourier series, Compositio Mathematica, vol. 7 (1939), pp. 112-122.

7. P. Turán, Über die montone Konvergenz der Cesàro-Mittel bei Fourier-und Potenzreihen, Proceedings of the Cambridge Philosophical Society, vol. 34, Part II (1938), pp. 134-143.

8. A. Zygmund, Trigonometrical Series, 1935.

University of Cincinnati,

Cincinnati, Ohio 